\title{
Radial Nerve Compression Caused by Traumatic Fat Necrosis
}

\author{
Hee Jong Hwang, Gwang Soo Lee, Hyung Ki Park, Jae-Chil Chang \\ Department of Neurosurgery, Soonchunhyang University Seoul Hospital, Soonchunhyang University College of Medicine, \\ Seoul, Republic of Korea
}

Corresponding author: Gwang Soo Lee

Department of Neurosurgery, Soonchunhyang University Seoul Hospital, Soonchunhyang University College of Medicine, 59, Daesagwan-ro, Yongsan-gu, Seoul 04401, Republic of Korea

Tel: $+82-2-709-9268$

Fax: $+82-2-792-5976$

E-mail: lgs790411@gmail.com

Received: April 9, 2017

Revised: June 27, 2017

Accepted: July 24, 2017
This report describes radial neve compression due to posttraumatic mass in the subcutaneous layer of an arm. Generally, these posttraumatic masses are mostly hematomas that develop from injured areas. The patient complained about motor weakness in hand and a tingling sensation of forearm. So we performed surgical resection and biopsy of the mass. The pathologic diagnosis was fat necrosis unlike our impression. Usually, the most common complaints of posttraumatic fat necrosis are a palpable mass and pain. In this case, the fat necrosis compressed the radial nerve and induced neurological symptoms.

Key Words: Injuries; Fat necrosis; Radial nerve

\section{INTRODUCTION}

In general, traumatic peripheral nerve injury (PNI) is more common in the upper limb ${ }^{5)}$. From $1 \%$ to $2 \%$ of patients with extremity trauma patients have injuries involving peripheral nerve ${ }^{9)}$. PNI can be an important cause of morbidity and disability. So, when evaluating patients with limb trauma, it is important to identify the cause of neurological symptoms. Posttraumatic mass of the forearm is a relatively frequent complication due to trauma in soft tissue. These posttraumatic masses can cause neurological symptoms by compressing the peripheral nerves. Posttraumatic masses include hematoma and lymphangioma, and neuroma in forearm. But, the mass was encapsulated by fat necrosis in our case.

Fat necrosis may appear as a palpation of a lump, local depression, and discoloration of the skin, and it causes localizing pain. There were a few reports of neurological symptoms due to fat necrosis. In our case, the patient complained of neurological symptoms such as weakness.

\section{CASE REPORT}

A 57-year-old female, patient was injured by a golf ball in the left arm about 1 year ago. Since then, she felt weakness in the left arm. She underwent physical therapy in the 1st month at another hospital. After this therapy, patient's weakness of elbow and wrist flexor muscle was improved from motor grade three to four. But after 8 months, a palpable mass appe- ared in the left upper arm of the patient. When this mass was stimulated, she complained about tingling sensation on posterior side of forearm from elbow to thumb. It was the area of radial nerve innervation. Also, she complained of motor weakness of the thumb because she could not complete extension. Another symptom was a tremor during wrist extension.

The patient underwent ultrasonography (US) because she underwent magnetic resonance imaging (MRI) in the 2 month of trauma at another hospital (Fig. 1). In this image, the mass $(6 \times 9 \mathrm{~mm})$ was observed on the left arm from lateral aspect. It's a high signal on T2 MRI image, and is hypoechoic lesion on US. Margins were indistinctive with unclear fluids and blood supply was not increased in doppler images. It was depressed by this hypoechoic mass that small hypoechoic areas separated by hyperechoic septae in the US image. This areas was innervated by radial nerve. Electromyography (EMG) \& Nerve conduction study (NCV) were performed to verify any neuronal damage. The tests showed that were the left ulnar \& superficial radial nerve were damaged in mid-arm level.

We needed to determine whether the neurological symptoms that the patient complained of could be treated surgically ${ }^{2}$. We rescaned MRI before the surgery (Fig. 2) and discorved about $9 \mathrm{~mm}$ sized lesionon lateral aspect of subcutaneous fat layer of left arm which was not evident from previous MRI.

Based on history, clinical features, and US findings, a traumatic hematoma was strongly suspected. So we planned a mass resection. The patient was placed in supine position and the left shoulder abduction, the elbow pronation, and the US were used, respectively, to confirmed mark the mass. We decided to apply the Intraoperative monitoring (IOM) with a motor 


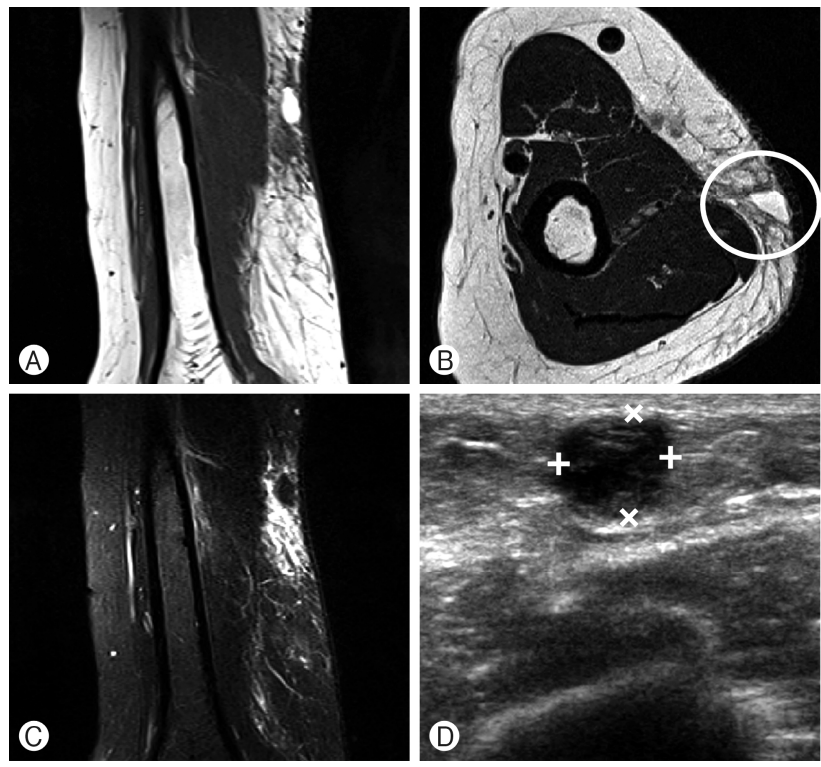

Fig. 1. Magnetic resonance imaging (MRI) and ultrasonography (US) images are taken in the second month at trauma. (A, B) There is a swelling of subcutaneous fat layer, lateral aspect of mid-portion of left upper arm with extension into intermuscular fascia surrounded by a hypointense rim. It is a well-defined mass of fat signal intensity (SI) (T1 weighted MRI). (C) The lesion represents a low SI surrounded by an isointense rim (T2 weighted MRI). (D) We could see a hypoechoic mass the size of $9 \mathrm{~mm}$. It is a well-defined oval shaped mass (US images). (B, D) It appears to be a radial nerve that was hypoechoic area with hyperechoic line in US image (white circle).

evoked potential (MEP) check to prevent any nerve damage during the operation.

During dissection of the mass using a microscope, we observed a drainage of yellowish fluids (Fig. 3). We performed curettage including surrounding fat tissue before ending the operation. However, radial nerve was not directly observed in the layer where surgery was performed. The radial nerve was not in direct contact with the mass and was indirectly pressured by the mass.

\section{RESULTS}

After the surgery, the motor grade of thumb extension improved from four to four positive and the tingling sensation was reduced. The tremor that occurred during wrist extension remained. The patient visited the outpatient department a 2 weeks after discharge, additional follow-up was stopped because the patient's symptoms were almost normal and the tremor disappeared. The histopathological examination of the mass revealed that was to be an encapsulated fat necrosis.

\section{DISCUSSION}

PNI frequently occurred in the upper limb than in the lower

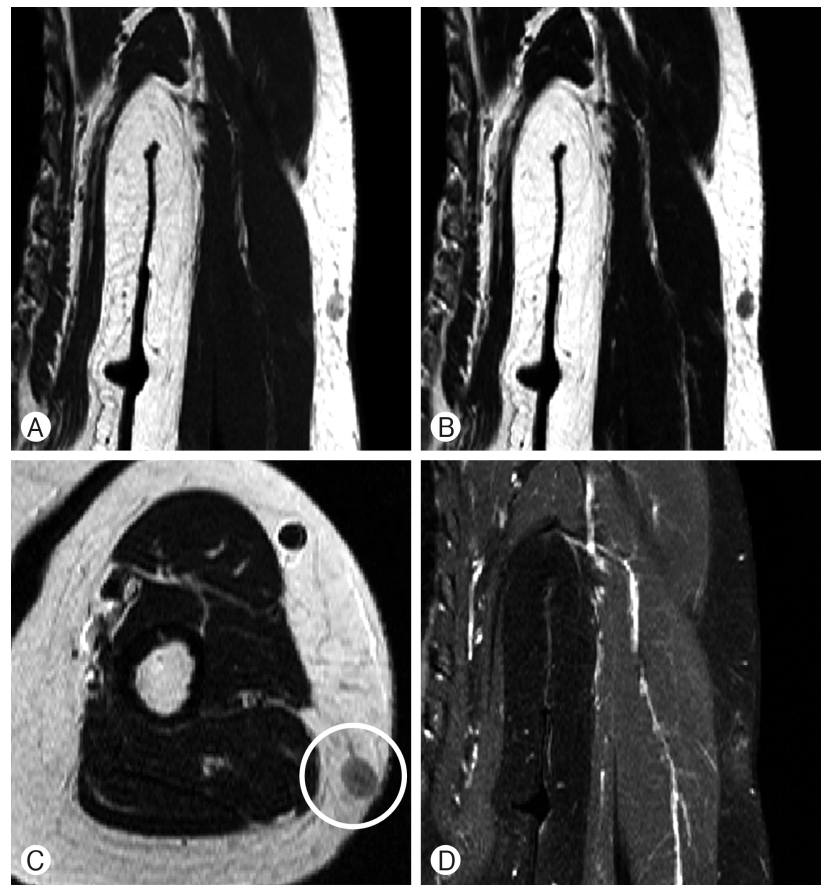

Fig. 2. There are left humerus was scaned by magnetic resonance imaging (MRI) after 1 years from previous MRI scan. (A-C) Disappearance of swelling of subcutaneous fat layer, lateral aspect of midportion of left upper arm with extension into intermuscular fascia of lateral aspect of upper arm muscles. There is about $9 \mathrm{~mm}$ sized $\mathrm{T} 1$ (A) slight high and T2 (B) heterogeneous low signal intensity (SI) lesion in lateral aspect of subcutaneous fat layer of left upper arm. (C) Radial nerve is stimulated by fat necrosis when compressing this mass (white circle). (D) Mass of fat $\mathrm{SI}$ is surrounded by hypointense rim capsule (gadolinium-enhanced $\mathrm{T} 1$ ).
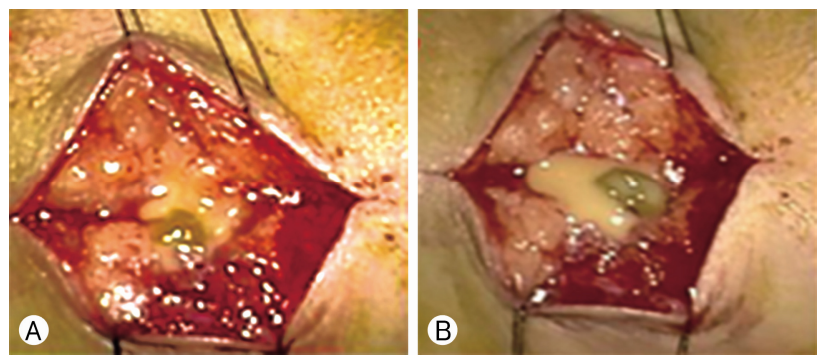

Fig. 3. This is a microscopic photograph at the time of surgery. (A) It was a soft and encapsulated mass. (B) When the mass was excised, it was gushed out yellowish liquid. So this liquid was cultured, but it was not pus and there was no growth. This soft tissue is mature fat tissue with fat necrosis.

$\operatorname{limb}^{1)}$. The most frequently injured nerve was the radial nerve ${ }^{9)}$. Some common causes of nerve compression symptoms were considered as tumor, lipoma, iatrogenic damage, and trauma. Most common cause is trauma. These posttraumatic masses can cause neurological symptoms by compressing the peripheral nerves. Post-traumatic masses include hematoma and lymphangioma ${ }^{6}$, neuroma in forearm. But, In this case, the mass was 
encapsulated by fat necrosis. So we wondered why we had diagnosed the mass as a hematoma, not a fat necrosis.

Fat necrosis is a benign entity frequently presenting itself as a palpable mass within the subcutaneous tissues. Posttraumatic fat necrosis can occur following a trauma, surgery, and any procedure including injections. It is more prevalent in women and usually appears on arms, breasts, thighs and buttocks $^{7)}$. Fat necrosis may appear as a palpable mass, local depression, discoloration of the skin, and it causes localizing pain.

Fat necrosis may present itself as subcutaneous nodules, so-called encapsulated fat necrosis ${ }^{2)}$. A variety of causes of fat necrosis has been reported, including trauma, collagen vascular disease, myeloproliferative disorders, and complications of pancreatic disorders such as in disseminated fat necrosis ${ }^{3)}$.

A traumatic event produces humoral factors which induce pre-adipocytes to mature. Thus producing an unencapsulated mass. It is presumed to be the mechanism of posttraumatic fat necrosis development. In trauma related cases, fat necrosis occurs in subcutaneous adipose tissues overlying pressure points or in the areas subject to trauma. Even in our case, fat necrosis seems to have occurred in the same way. In addition, herniation of fat through a fascial tear may be another cause of traumatic fat necrosis. The pathogenesis of subcutaneous fat necrosis is somewhat controversial ${ }^{1)}$. It seems to be related to rapid vascular insufficiency and subsequent fibrous capsule formation $^{8)}$. Local or systemic events, such as pancreatitis, causing a compromise in the blood supply of the subcutaneous tissues result in necrosis of adipose tissue and induce an inflammatory response. Fat necrosis has been reported in patients with collagen vascular diseases, such as systemic sclerosis, likely resulting from small vessel-related ischemia in the subcutaneous adipose. However, in our case, the patient developed fatty necrosis even without systemic disease which was an uncommon case.

The best tools for diagnosing necrosis are MRI and US. The computed tomography image of fat necrosis in the extremities has been poorly described ${ }^{3)}$. On MRI, Axial unenhanced T1-weighted image (WI) shows well-defined lobulated intramuscular mass of fat signal intensity (SI). The lesion may have or lack a pseudocapsule with hypointense rims. Fibrous or granulation tissue comprising the pseudocapsule surrounding or within the lesion is low in SI on T1 and fast spin echo (FSE) T2-WI and intermediate to high SI on the short tau inversion recovery (STIR) sequence, corresponding to reactive fibrous tissues. Fat necrosis has a similar imaging appearance as mature adipose tissue. However, difference forms of fat necrosis were seen in the lipomas and atypical lipoma on MRI ${ }^{3)}$. Therefore, additional US was necessary for accurate diagnosis. US can assess the nerves and may detect any morphological changes of the nerves. US can also reveal the causes of nerve compression when there are structural abnormalities or space-occupying lesions are present. The most common US finding of the compression neuropathy is a nerve swelling proximal to the compression site ${ }^{4)}$.

US image displays a hypoechoic appearance in most fat necrosis. But the sonographic appearance of isolated fat necrosis of the trunk in extremities has not been well characterized in the literature ${ }^{10)}$. Also, its US findings were very variable. Walsh et al. ${ }^{10)}$ report that echogenicity was $40 \%$ isoechoic, $60 \%$ hyperechoic, $80 \%$ heterogeneous, and 20\% homogeneous. A completely surrounding hypoechoic halo was seen in $40 \%$. The area of fat necrosis was poorly defined in $60 \%$ and well defined in 40\%, showing a mass effect in $60 \%$. They also report that the sonographic appearance of extremity fat necrosis includes a well-defined isoechoic mass with hypoechoic halo and a poorly defined hyperechoic abnormality within the subcutaneous fat. And, these were quite different from our ultrasonic findings.

We could not distinguish between fat necrosis from other mass due to nerve compression symptoms despite performing MRI and ultrasound. Also Posttraumatic fat necrosis has not been reported to cause neurological symptoms by compressing radial nerves.

If the neurological symptoms of radial nerve are suspected after trauma, precise lesion detection is needed using ultrasound and MRI as well as EMG \& NCV. Despite these advanced softtissue imaging devices, an accurate diagnosis remains absent, and further surgical exploration is necessary for better diagnosis and treatment.

\section{CONCLUSION}

This is the rare case reporting an encapsulated fat necrosis that presented posttraumatic neurological symptoms on upper limb.

\section{CONFLICTS OF INTEREST}

No potential conflict of interest relevant to this article was reported.

\section{REFERENCES}

1. Aust MC, Spies M, Kall S, Vogt PM: Diagnosis and treatment of posttraumatic pseudolipomas. A retrospective analysis. Unfallchirurg 109:948-955, 2006

2. Burkholz KJ, Roberts CC, Lidner TK: Posttraumatic Pseudolipoma (Fat Necrosis) Mimicking Atypical Lipoma or Liposarcoma on MRI. Radiol Case Rep 2:56-60, 2007

3. Chan LP, Gee R, Keogh C, Munk PL: Imaging features of fat necrosis. AJR Am J Roentgenol 181:955-959, 2003

4. Choi SJ, Ahn JH, Ryu DS, Kang CH, Jung SM, Park MS, et al.: Ultrasonography for nerve compression syndromes of the upper extremity. Ultrasonography 34:275-291, 2015 
5. Griffin MF, Malahias M, Hindocha S, Khan WS: Peripheral nerve injury: principles for repair and regeneration. Open Orthop J 8:199-203, 2014

6. Guruswamy M, Ahluwalia RS, Shanker VS: Post-traumatic lymphangioma of the forearm in a young adult male. Grand Rounds 6:22-24, 2006

7. Haikin Herzberger E, Aviner S, Cherniavsky E: Posttraumatic fat necrosis presented as cellulitis of the leg. Case Rep Pediatr 2012:672397, 2012

8. Hurt MA, Santa Cruz DJ: Nodular-cystic fat necrosis. A reeva- luation of the so-called mobile encapsulated lipoma. J Am Acad Dermatol 21:493-498, 1989

9. Moore AM, Wagner IJ, Fox IK: Principles of nerve repair in complex wounds of the upper extremity. Semin Plast Surg 29: 40-47, 2015

10. Walsh M, Jacobson JA, Kim SM, Lucas DR, Morag Y, Fessell DP: Sonography of fat necrosis involving the extremity and torso with magnetic resonance imaging and histologic correlation. J Ultrasound Med 27:1751-1757, 2008 